\title{
La pteridoflora (helechos y licófitos) del Espacio Natural Sierra de las Nieves (Málaga, España)
}

Federico Casimiro-Soriguer Solanas, Andrés Vicente Pérez Latorre \& Baltasar Cabezudo

Área de Botánica. Universidad de Málaga. España.

\section{Correspondencia.}

F. Casimiro-Soriguer

e-mail: fedeque@hotmail.com

Recibido: 24 junio 2020

Aceptado: 13 julio 2020

Publicado on-line: 22 julio 2020

Editado por: Marta Recio Criado

\begin{abstract}
Resumen
Se presenta en este trabajo un catálogo de los helechos y licófitos del Espacio Natural Sierra de las Nieves, territorio localizado al sur de la Península Ibérica (Málaga, España) y del que forman parte el Parque Natural Sierra de las Nieves y el propuesto como Parque Nacional Sierra de las Nieves. Se han localizado 38 taxones pertenecientes a 10 familias. De los tres subsectores biogeográficos que conforman el espacio natural, los que presenta mayor diversidad de taxones son el Marbellí con 24 taxones y el Rondense con 21 y el de menor diversidad el Bermejense con 16. Del total de taxones 7 son comunes a todos los subsectores, siendo exclusivos 8 para el subsector Marbellí, 9 para el Rondense y 4 para el Bermejense. Las familias más importantes en diversidad son Aspleniaceae y Pteridaceae con 12 taxones cada una. El resto de las familias oscilan entre 1 y 3 taxones. De los 38 taxones consideramos 14 como muy raros en el espacio natural y como muy frecuentes 10. Solo dos taxones de los localizados se encuentran amenazados como VU (Vulnerables) a nivel de Andalucía y solo uno está protegido en la legislación andaluza como RPE (Régimen de Protección Especial).
\end{abstract}

Palabras claves. Parque Nacional, Parque Natural, helechos, licófitos, Andalucía, Serranía de Ronda.

\begin{abstract}
Pteridophyte catalogue (ferns and lycophytes) from Sierra de las Nieves Natural Area (Malaga, Spain)

A catalogue of ferns and lycophytes has been made from the Sierra de las Nieves Natural Area. It is a territory located in southern Iberian Peninsula (Malaga province, Spain) and it is composed of Sierra de las Nieves Natural Park and a surrounding area proposed as National Park. 38 taxa belonging to 10 families have been identified. Of the three biogeographic subsectors that make up the natural area, those with the greatest diversity of taxa are the Marbella subsector with 24 taxa and the Ronda subsector with 21, and the least diverse is the Bermeja subsector with 16. Of the total of taxa, 7 are common to all subsectors. 8 are exclusive for the Marbella subsector, 9 for Ronda and 4 for Bermeja. The most important families in diversity are Aspleniaceae and Pteridaceae with 12 taxa each. The rest of the families range from 1 to 3 taxa. Of the 38 taxa, we consider 14 to be very rare in the natural area and 10 as very frequent. Only two of the catalogued taxa are considered as threatened (vulnerable) in Andalusia and only one is protected under Andalusian legislation (Regime of Special Protection).
\end{abstract}

Key words. National Park, Natural Park, ferns, Andalusia, Serrania de Ronda

\section{Introducción}

El Espacio Natural Sierra de las Nieves, con 29580 ha, se localiza en la Serranía de Ronda (Málaga, España) y está formado por el actual Parque Natural Sierra de las Nieves y la propuesta de Parque Nacional Sierra de las Nieves. Del territorio de este espacio natural, enclavado en la Cordillera Bética Occidental, forman parte 3 sectores biogeográficos (Figura 1): Aljíbico (subsector Marbellí) (litológicamente formado por micaesquistos y gneises), Bermejense (subsector Bermejense) (peridotitas) y Rondeño (subsector Rondense) (calizas y dolomías) (cf. Pérez Latorre et al., 2019). Los términos municipales presentes en la zona de estudio son: Benahavís, El Burgo, Igualeja, Istán, Monda, Parauta, Ronda, Tolox y Yunquera (figura 1 in Guerra et al., 2018). 
El conjunto presenta una elevada heterogeneidad ambiental tanto litológica como térmica, con termotipos desde el termomediterráneo al oromediterráneo, y altitudes entre los 200 y 1919 msnm, perteneciendo al macrobioclima Mediterráneo (Rivas Martínez, 2011), lo que contribuye a su importante diversidad vegetal (Pérez Latorre et al., 1998). No conocemos ningún trabajo previo centrado exclusivamente en la flora pteridológica de este espacio natural ni de sus áreas limítrofes. Algunos datos aparecen en los trabajos sobre pteridófitos de Andalucía (Salvo \& Cabezudo, 1984), los inventarios fitosociológicos publicados por Pérez Latorre et al. (1998) en la Serranía de Ronda (sector Rondeño) y en el informe correspondiente a la Junta de Andalucía de Cabezudo et al. (1998), así como en el cercano Parque Natural de los Alcornocales (sector Aljíbico) por Guerra et al (2003) y en las sierras ultramáficas malagueñas por Pérez Latorre et al. (2013).
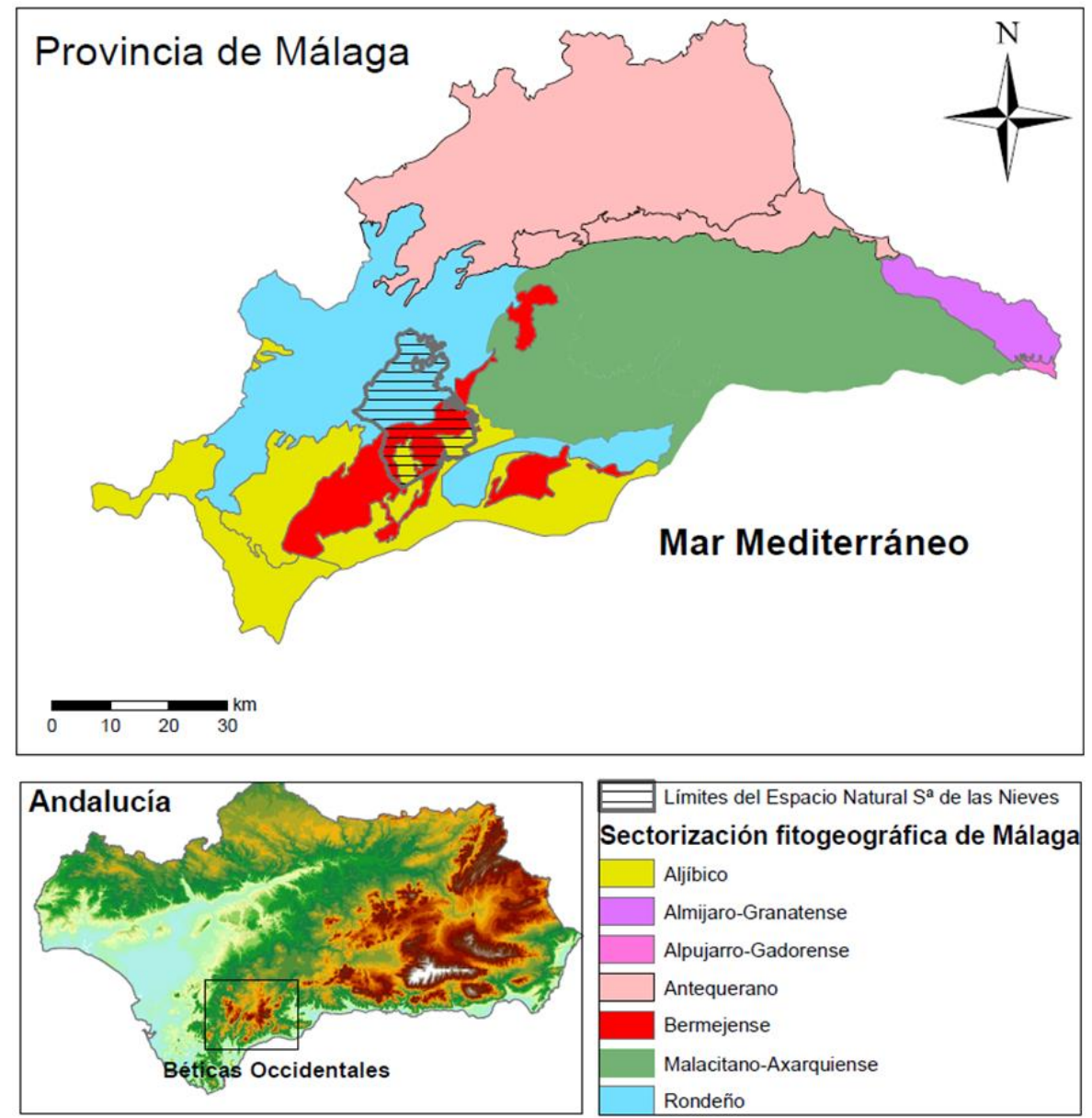

Figura 1. Situación geográfica en el sur de la Península lbérica y sectorización biogeográfica del Espacio Natural Sierra de las Nieves.

Figure 1. Geographical location in the southern Iberian Peninsula and biogeographic sectorization of the Sierra de las Nieves Natural Area.

\section{Metodología}

La mayor parte del material utilizado para este trabajo fue recolectado por los autores en los tres subsectores indicados durante los años 2017 al 2020, material que una vez preparado, identificado e informatizado, fue depositado en el herbario MGC de la Universidad de Málaga. Se ha empleado también todo el material depositado, con anterioridad a este trabajo, en el mencionado herbario MGC.

Para la identificación del material recolectado nos hemos basado fundamentalmente en las obras de Castroviejo et al. (1986), Cabezudo \& Salvo (1987), Salvo (1990), Cabezudo \& Trigo (2004) y Cabezudo (2011). Para el complejo de Asplenium trichomanes hemos recurrido a Pangua et al. (1989), Sáez (2000) y (Ekrt \& Stech, 2008). Para los comentarios corológicos hemos consultado, aparte de las obras anteriores, a Moreno et al. (2015) y la base de datos ANTHOS (www.anthos.es). Para las categorías de amenaza hemos utilizado el trabajo de Cabezudo \& Talavera (2005).

Para la sistemática general del grupo seguimos la síntesis que para la Península lbérica recoge Gabriel y Galán et al., (2018), basada en el PPG1. Para el género Cheilanthes adoptamos la propuesta de Fraser-Jenkins et al. (2017), por lo que todas las especies de la zona englobadas anteriormente en este género tienen ahora como nombre genérico correcto Oeosporangium, criterio ya empleado por otros autores ibéricos (Sáez et al., 2020).

Teniendo en cuenta la importancia de la hibridación en los procesos de especiación en los helechos, hemos incluido también en el catálogo 
aquellos híbridos de los que tenemos constancia en el territorio, aunque es probable que sean más numerosos, sobre todo en los géneros Asplenium y Oesporangium, habida cuenta de la diversidad taxonómica de ambos géneros en la zona.

El catálogo está dividido en licófitos (Cl. Lycopodiopsida) y monilófitos o helechos (Cl. Polypodiopsida) y ordenado luego por orden alfabético de familias y géneros. Para cada taxón se recoge la siguiente información: subsectores biogeográficos donde está presente. (Marbellí, Rondense, Bermejense); abundancia en la zona de estudio ( muy rara, rara, ocasional frecuente), terminología empleada por Blanca et al., (2011); hábitat típico en la zona de estudio (roquedos, taludes, suelos de sotobosques, suelos más o menos encharcados, paredes y fondos de simas); principal clase fitosociológica en la que se encuadra; rango altitudinal; pliegos testigos (se incluye un pliego testigo por término municipal donde se ha recolectado el taxón). Cuando se ha creído necesario se han incluido referencias corológicas previas y/o comentarios taxonómicos, así como las sinonimias más habitualmente empleadas.

Todos los pliegos recolectados en los años 2017 al 2020 se encuentran depositados en el herbario MGC y georrefrenciados al menos en cuadriculas UTM de $1 \times 1 \mathrm{~km}$.
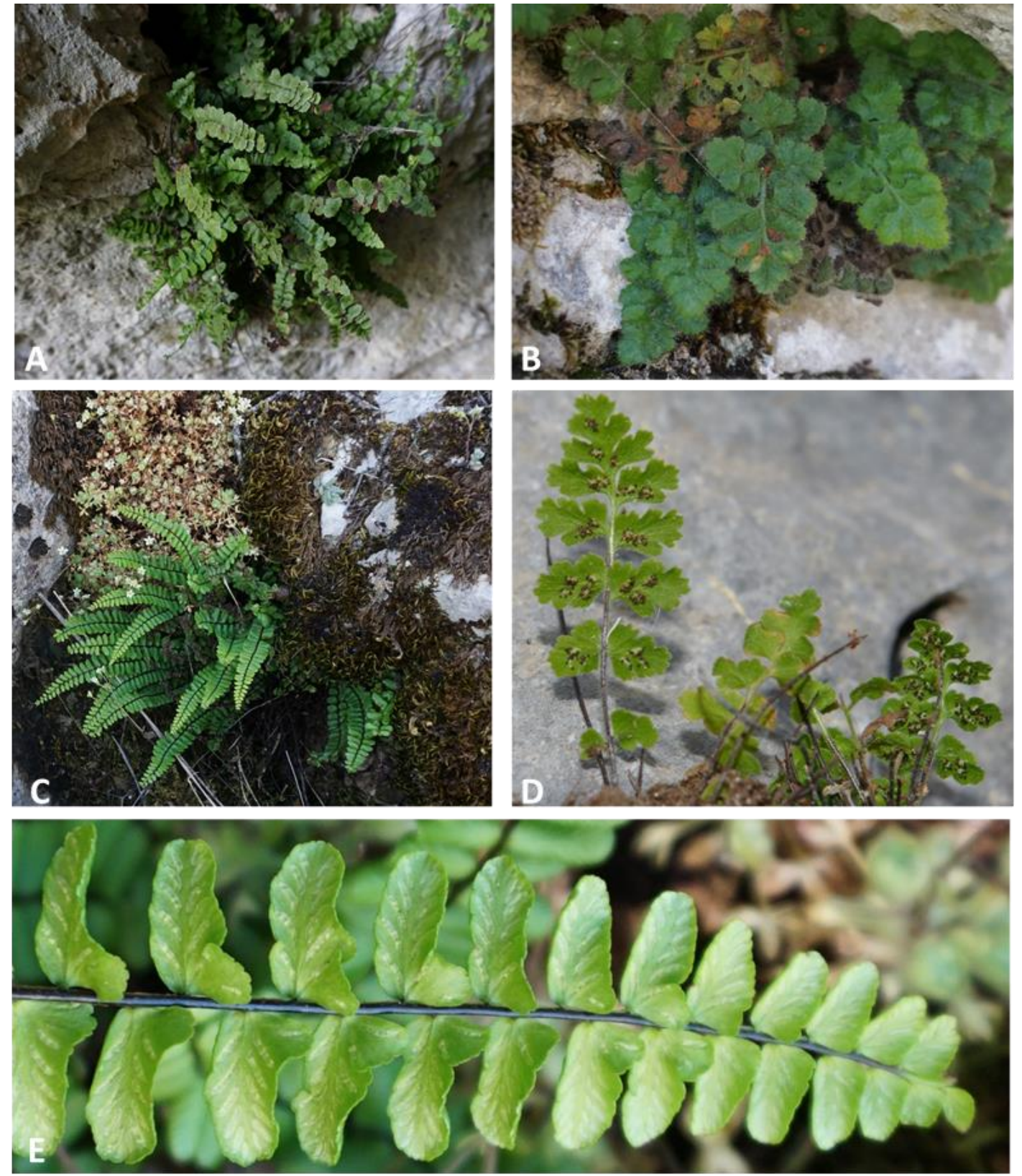

Figura 2. Taxones del género Asplenium de óptimo rondense, todos rupícolas y calcícolas: A) $A$. jessenii (Pilar de Tolox, $1700 \mathrm{msnm}$.); B) A. hispanicum (Cortijo de Bolina, $1165 \mathrm{msnm}$ ); C) A. azomanes (Cortijo de Bolina, $1165 \mathrm{msnm}$ ); D) A. petrarchae (Cortijo de Bolina, $1165 \mathrm{msnm}$ ); E) A. azomanes, detalle del fronde, nótese la neta aurícula de las pinnas que aloja un soro en distinta disposición al resto. Figure 2. Taxa of Asplenium genus typical of rondense subsector, all of them rupicolous and calcicolous: A) A. jessenei (Pilar de Tolox, 1700 msnm.); B) A. hispanicum (Cortijo de Bolina, $1165 \mathrm{msnm}$ ); C) A. azomanes (Cortijo de Bolina, $1165 \mathrm{msnm}$ ); D) A. petrarchae (Cortijo de Bolina, $1165 \mathrm{msnm}$ ); E) A. azomanes, detail of the frond, note the clear auricle of the pinnae that has a soro in a different arrangement from the rest. 


\section{Resultados}

\section{Cl. Lycopodiopsida}

\section{Fam. Selaginellaceae}

1.- Selaginella denticulata (L.) Spring in Flora 21: 149 (1838).

Marbellí, Bermejense y Rondense. Frecuente. Taludes umbríos, termófila. AnomodontoPolypodietea. 400-900 msnm. Benahavís (MGC 84690), El Burgo (MGC 89531), Istán (MGC 87425), Monda (MGC 3595), Tolox (MGC 85457).

\section{Cl. Polypodiopsida}

\section{Fam. Aspleniaceae}

2.- Asplenium adiantum-nigrum L., var. silesiacum (Mllde) Viane \& Reichst. in Candollea 49(1): 283 (1994)

[= var. corunnense H. Christ in Bull. Acad.Int. Géogr. Bot. 13(172): 78 (1904)].

Bermejense. Raro. Fisuras de roquedos peridotíticos generalmente en umbrías en el ámbito de pinares. Asplenietea trichomanis. 900-1450 msnm. Considerado como serpentinófito obligado (Pérez Latorre et al., 2013). Benahavís (MGC 49344), Igualeja (MGC 86580), Istán (MGC 86297), Parauta (MGC 53753), Tolox (MGC 86482).

3.- Asplenium billotii F.W. Schultz in Flora (Regensburg) 28: 738(1845) (Figura 3 C)

Marbellí. Ocasional. Casi amenazada (NT). Legalmente protegida (RPE). Taludes y roquedos umbrosos en el ámbito de alcornocales y quejigares. Phagnalo-Rumicetea. 200-1350 msnm. Benahavís (MGC 78747), Igualeja (MGC 60699), Istán (MGC89610), Parauta (MGC 91348), Tolox (MGC 88251).

\section{4.- Asplenium ceterach L., Sp. PI. 1080 (1753)}

[= Ceterach officinarum Willd., Anleit. Selbststud. Bot.: 578 (1804)]

Marbellí, Bermejense y Rondense. Frecuente. Taludes, roquedos y muros sobre todo tipo de substratos. Asplenietea trichomanis. 200-1800 msnm. El Burgo (MGC 87461), Istán (MGC 91396), Monda (MGC 3585), Parauta (MGC 89321), Ronda (MGC 89250), Tolox (MGC 87448), Yunquera (MGC 85953).

5.- Asplenium hispanicum (Cosson) Greuter \& Burdet in Willdenowia 10: 17 (1980) (Figura 2 B)

[= Pleurosorus hispanicus (Cosson) Morton in Bull. Soc. Bot. France 106: 233 (1959)]

[= Asplenium subglandulosum subsp. hispanicum (Coss.) Salvo et al. in Candollea 37: 481 (1982)]

Rondense. Muy raro. Rupícola en roquedos umbríos en situaciones protegidas, sobre calizas y dolomías. Asplenietea trichomanis. 1200-1350 msnm. El Burgo (MGC 92863, MGC 92866).
6.- Asplenium onopteris L. in Sp. PI.: 1081 (1753) (Figura 3 B)

Marbellí. Frecuente. Sotobosque de alcornocales y quejigares. Quercetea ilicis. 300-1300 msnm. Benahavís (MGC 85101), Igualeja (MGC 22951), Istán (MGC 84794), Monda (MGC 6486), Ronda (MGC 33318), Tolox (MGC 89335), Yunquera (MGC 8384).

7.- Asplenium petrarchae (Guerin) DC. in Lam. \& DC., FI. Franc. Ed. 3, 5: 238 (1815) (Figura 2 D)

Rondense. Muy raro. En roquedos expuestos. Asplenietea trichomanis. 620-1100 msnm. Tolox (MGC 89629), El Burgo (MGC 92869).

A falta de un estudio morfométrico de las esporas del material recolectado, que permita dilucidar a que subespecie pertenece, incluimos dicho material en la especie en sentido amplio.

8.- Asplenium ruta-muraria L., Sp. PI.: 1081 (1753) (Figura 4 C)

Rondense. Muy rara. Roquedos calizos y dolomíticos umbríos. Asplenietea trichomanis. 1600 1800 msnm. Tolox (MGC 44986).

9.- Asplenium scolopendrium L., Sp. PI.: 1079 (1753)

[= Phyllitis scolopendrium (L.) Newman, Hist. Bri. Ferns ed. 2: 10 (1844)]

Rondense. Muy rara. Rupícola umbrófila. En simas calizas del piso supra-oromediterráneo. Solo conocemos una población con 3 individuos en el fondo de una sima en la meseta del Quejigal de Tolox. 1700 msnm. Tolox (MGC 87436). Las únicas citas previas de esta especie para la zona son de Laza (1940) donde ya se comenta su rareza en la zona.

Asplenium grex trichomanes L., Sp. Pl.: 1080 (1753)

De la complejidad de este grupo dan fe la disparidad de criterios al interpretar morfológicamente los distintos taxones citados en Europa (Pangua et al.,1989; Sáez, 2000; Ekrt \& Stech, 2008). Aunque este complejo de taxones con distintos niveles de ploidía son muy similares morfológicamente parecen representar especies netamente diferenciadas genéticamente (Liu et al., 2018). Nosotros, a falta de un estudio de mayor envergadura del agregado, hemos optado por seguir principalmente a Sáez (2000) para nombrar los 4 grupos de plantas que hemos podido diferenciar por sus caracteres morfológicos:

10.- Asplenium azomanes Rosselló, Cubas \& Rebassa in Rivasgodaya 6: 115-128 (1991) (Figuras $2 \mathrm{C}, 2 \mathrm{E})$

[= Asplenium trichomanes L. subsp. coriaceifolium H. Rasbach, K. Rasbach, T. Reichstein \& H. W. Bennert in Willdenowia 19: 471 (1990)]

Rondense. Raro. Rupícola en roquedos calizos y dolomíticos. Asplenietea trichomanis. 700 -1300 
msnm. El Burgo (MGC 92867), Parauta (MGC 89987), Tolox (MGC 87732).

Este taxón englobaría a plantas rupícolas, con frondes no aplicadas al substrato, y pinnas asimétricas en la base. Las pinnas medias y superiores con una aurícula muy desarrollada en su parte basal, aurícula que aloja en algunas de esas pinnas un soro dispuesto asimétricamente al resto.

11.-Asplenium csikii Kummerle \& Andrásovszky in
Magyar Bot. Lapok. 21: 3 (1922)

$[=A$. trichomanes subsp. pachyrrachys (Christ) Lovis \& Reichst. in Willdenowia 10: 18 (1980)]

Rondense. Muy raro. Roquedos calizos y dolomíticos. Asplenietea trichomanis. 1700-1800 msnm. Tolox (MGC 45735).

Este taxón engloba a las plantas rupícolas, con frondes aplicadas al substrato, con pinnas de base simétrica y al menos el doble más largas que anchas, frecuentemente con 2 aurículas en la base.
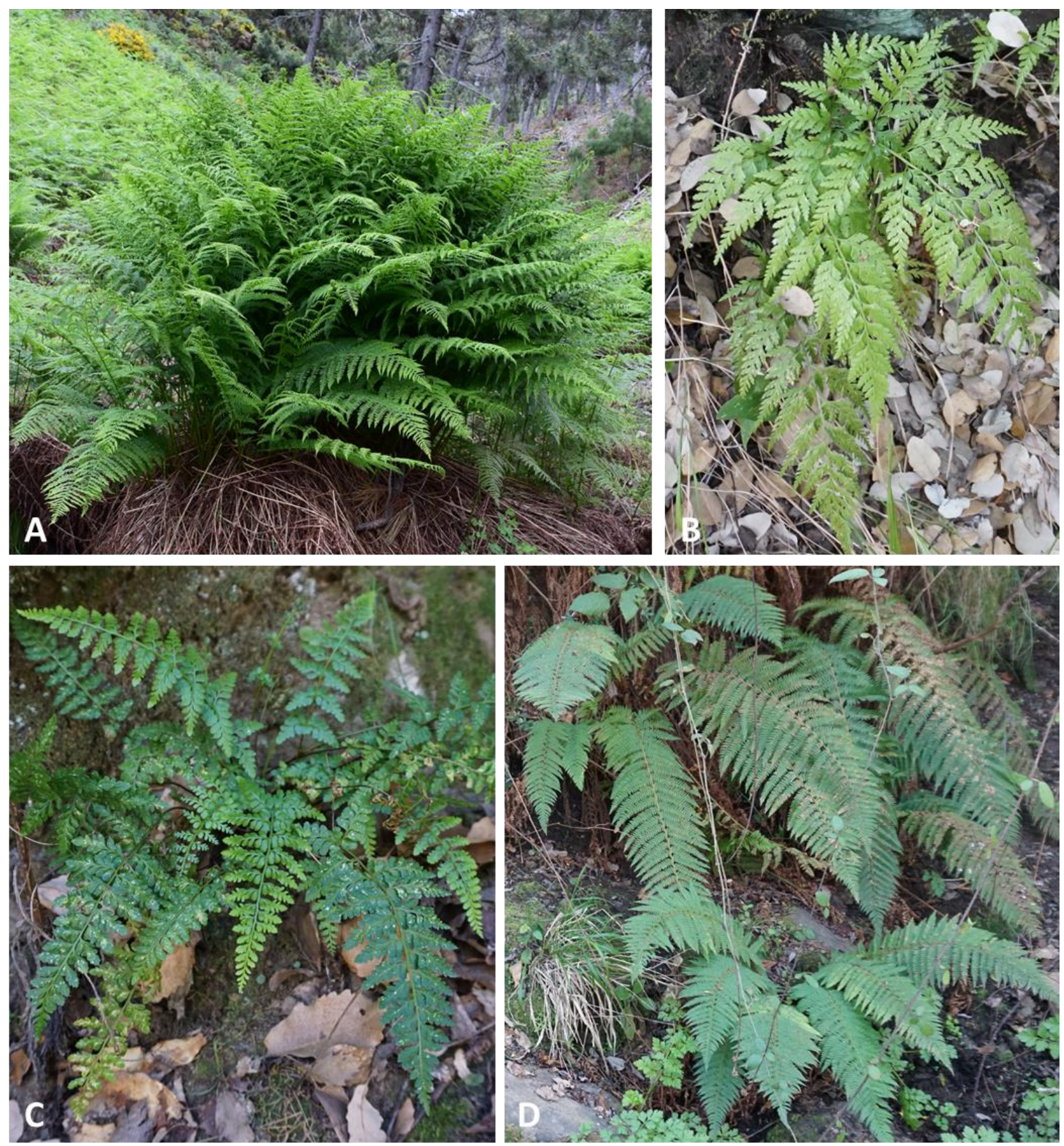

Figura 3. Helechos de óptimo aljíbico en la zona de estudio: A) Athyrium filix-femina (Peñón del Robledal, 1350 msnm.); B) Asplenium onopteris (La Máquina, 250 msnm.); C) Asplenium billotii (Peñón del Robledal, 1350 msnm.); D) Polystichum setiferum (Barranco de la Cuerna, $550 \mathrm{msnm}$.). Figure 3. Ferns typical of Aljibico sector in estudy area: A) Athyrium filix-femina (Peñón del Robledal, $1350 \mathrm{msnm}$.); B) Asplenium onopteris (La Máquina, $250 \mathrm{msnm}$.); C) Asplenium billotii (Peñón del Robledal, 1350 msnm.); D) Polystichum setiferum (Barranco de la Cuerna, 550 msnm.) 
12.- Asplenium trichomanes L. subsp. quadrivalens D.E. Meyer in Ber. Deutsch. Bot. Ges. 74: 456 (1962)

Marbellí, Bermejense y Rondense. Frecuente. Roquedos y taludes, normalmente en umbrías, sobre todo tipo de substratos. Asplenietea trichomanis. 250-1700 msnm. Benahavís (MGC 89437), El Burgo (MGC 87462), Igualeja (MGC 22910), Istán (MGC 89849), Parauta (MGC 89987), Ronda (MGC 87510), Tolox (MGC 86998), Yunquera (MGC 87442).

Es el taxón más extendido y a él pertenecen las plantas con frondes no aplicadas al substrato, y con pinnas asimétricas en la base y todos los soros dispuestos simétricamente.

13.- Asplenium jessenii H.M. Liu \& H. Schneid in PI. Syst. Evol. 304: (2018) (Figura 2 A)

[= Asplenium trichomanes subsp. hastatum (Christ) Jessen in Ber. Bayer. Bot. Ges. 65: 111 (1995)]

Rondense. Ocasional. Roquedos calizos 0 dolomíticos. Asplenietea trichomanis. 1000-1800 msnm. Ronda (MGC 89251), Tolox (MGC 91814), Yunquera (MGC 44977).

Adjudicamos a este taxón las plantas con frondes más o menos aplicadas al substrato, con pinnas imbricadas y simétricas en la base, de contorno triangular, de longitud y anchura similares o solo un poco más largas y más o menos biauriculadas en la base.

\section{Fam. Athyriaceae}

14.- Athyrium filix-femina (L.) Roth, Tent. Fl. Germ. 3: 65 (1799) (Figura 3 A)

Marbellí. Muy rara. Suelos con encharcamiento permanente. Localizada en la cuenca del río Guadaiza, donde aparece de forma dispersa, y en la cara norte del Peñón del Robledal donde forma una comunidad higrófila muy desarrollada en pequeños arroyos con agua permanente. Salici-Populetea. 400-1300 msnm. Benahavís (MGC 78323), Igualeja (MGC 86524).

La localidad del Peñón del Robledal (Igualeja) ya fue dada a conocer por Nieto et al. (1988), aunque indicando un bajo número de ejemplares, lo que no corresponde con las densas formaciones que hemos observado en la actualidad.

\section{Fam. Cystopteridaceae}

15.- Cystopteris dickieana R. Sim in Gard. Farmer's J. 2(20): 308 (1848).

Rondense. Ocasional. Muy raro en el Marbellí y Bermejense. Roquedos umbríos. Asplenietea trichomanis. 1250-1750 msnm. Igualeja (MGC 60698), Parauta (MGC 58697), Ronda (MGC 36194), Tolox (MGC 81084).

16.- Cystopteris diaphana (Bory) Blasdell in Mem. Torrey Bot. Club 21(4): 47 (1963)

[= Cystopteris viridula (Desv.) Desv. in Mém. Soc. Linn. Paris 6: 264(1827)]
Marbellí. Muy rara. Taludes rezumantes en sotobosques de quejigales. Adiantetea. $600 \mathrm{msnm}$. Solo conocemos una población, en la cuenca del río Verde, Arroyo de la Cruz. Istán (MGC 89850, 89851).

Según Murphy \& Rumsey (2005), C. viridula estaría englobado dentro de un más extendido geográficamente $C$. diaphana, como anteriormente sugerían Prada (1986) y Salvo (1990).

C. diaphana es una especie muy rara en Andalucía, localizada sobre todo en la Sierra Nevada granadina (Moreno Saiz et al., 2015), con poblaciones muy localizadas en la Sierra Norte onubense (Cabezudo et al., 1983) y el Valle del Genal en Málaga (Gavira \& Pérez Latorre, 2003), hasta ahora la única localidad para la provincia de Málaga. Aunque en la zona aljíbica de la zona estudiada no faltan ambientes propicios para el desarrollo de esta especie, solo hemos podido confirmar una población, lo que da idea de su escasez y exigencias ecológicas.

17.- Cystopteris fragilis subsp. fragilis (L.) Bernh. in Neues J. Bot. 1(2): 27 (1805).

Rondense. Ocasional. Roquedos umbríos. Asplenietea trichomanis. 1400-1800 msnm. Tolox (MGC 92573), Yunquera (MGC 21778), Ronda (MGC44984), Parauta (MGC 53754).

\section{Fam. Davalliaceae}

18.- Davallia canariensis (L.) Sm. in Mém. Acad. Roy. Sci. (Turin) 5: 414 (1793) (Fig. 5 B)

Marbellí. Muy rara. Amenazada (VU). Roquedos en valles encajonados, térmicos. AnomodontoPolypodietea. Aparece de forma muy localizada en la cuenca del río Guadaiza. 200-550 msnm. Benahavís (MGC 65778, MGC 78230).

Especie que en la Península lbérica se distribuye por la fachada atlántica, en el sur de la Península Ibérica se localiza fundamentalmente en las sierras aljíbicas con sus poblaciones finícolas hacia oriente en la provincia de Málaga, en el Valle del Genal (Nieto Caldera et al., 1987; Gavira \& Pérez Latorre, 2003), sierras bermejas (Salvo \& Cabezudo, 1984) y la cuenca del río Guadaiza, encontrada aquí por primera vez por Nieto Caldera en 1978 , siendo esta última población la más oriental de la Península Ibérica. Dudamos de una cita más oriental (Torrox, Málaga), recogida en Morales Abad (1990), en base a un pliego depositado en el herbario de Ginebra (G), recolectado en 1908 y puesta en duda por el mismo Morales Abad (1990).

\section{Fam. Dennstaedtiaceae (Incluye a Hypolepidaceae)}

19.- Pteridium aquilinum (L.) Kuhn in Kersten, Reis. Ost-Afr. 3(3): 11 (1879)

Marbellí, Bermejense y Rondense. Frecuente. Sotobosques y claros en suelos ácidos con cierta humedad y generalmente alterados por fuego o rozas. Cytisetea scopario-striatii. 250- $1375 \mathrm{msnm}$. Benahavís (MGC 84789), Igualeja (MGC 87364), 
Istán (MGC 92562), Parauta (MGC 91991), Tolox (MGC 88250), Yunquera (MGC 46767).

En la población del Peñón del Robledal (Igualeja) hemos localizado varios individuos con esporangios y esporas perfectamente desarrolladas.

\section{Fam. Dryopteridaceae}

20.- Dryopteris filix-mas (L.) Schott, Gen. Fil., tab. 9 (1834) (Figura 4 D)

Rondense. Muy rara. Amenazada (VU). Rupícola umbrófila en simas calizas del piso supraoromediterráneo. Querco-Fagetea. Solo hemos localizado una población en la entrada de una sima (Sima Honda) en la Meseta del Quejigal de Tolox. 1715 msnm. (MGC 66804, MGC 90795)

La única cita que conocíamos para la Sierra de las Nieves es la de Delgado \& Plaza (2010), referida a esta única población y basada en una recolección del año 2007. La población de la Sierra de las Nieves es la más meridional de Península lbérica, según recogen estos últimos autores. Hemos censado esta población que consta de 9 ejemplares que colonizan las repisas de entrada de la sima, así como las paredes verticales de los 20 primeros metros de la cavidad.

21.- Polystichum aculeatum (L.) Roth, Tent. Fl. Germ. 3: 79 (1799) (Figura 4 A)

[= Aspidium aculeatum (L.) Sw. in J. Bot. (Schrader) 1800 (2): 37 (1802)]

Rondense. Muy rara. Rupícola en simas calizas del piso supra- oromediterráneo. Querco-Fagetea. Solo conocemos una pequeña población en el fondo de una sima en el Puerto de los Pilones. 1750 msnm. Tolox (MGC 6373, MGC 92574).

Esta especie ya fue citada para la zona de estudio, recogiendo las citas de Haenseler y Prolongo, por Boissier (1839-1845, 2: 691) donde dice: "En fisuras profundas de la región alpina, en Sierra de la Nieve, $1800 \mathrm{msnm}$ ", la misma ecología y a una altitud similar que la de la única población que conocemos en la zona. Esta población fue localizada en el año 1980 (MGC 6373). Hemos censado 6 individuos sobre el suelo pedregoso del fondo de una pequeña sima de unos 10 metros de profundidad.

La población de la Sierra de las Nieves parece ser la más meridional de la especie en la Península Ibérica, ya que la cita de las sierras algecireñas (Cádiz) (Rivas Goday, 1968), se refiere con toda probabilidad a $P$. setiferum, muy abundante en la zona y más característica de los bosques de Quercus broteroi y $Q$. canariensis (Pérez Latorre et al., 1996; Guerra et al. 2003).

22.- Polystichum setiferum (Forssk.) Woyn. in Mitt. Naturwiss. Vereines Steiermark 49: 181 (1913) (Figura $3 \mathrm{D}$ ).

Marbellí. Muy rara. Casi amenazada (NT). Sotobosque de quejigales y lauredas en barrancos muy umbríos y térmicos. Quercetea ilicis. Solo conocemos una población en la cuenca del río Guadaiza, Cañada de la Cuerna. 550 msnm; Benahavís (MGC 29779, MGC 89456).

Esta especie fue localizada por primera vez en el año 1991 (MGC 29779) y, según indica la etiqueta del pliego testigo, la población se componía de unos 15 individuos. Hemos vuelto a localizar esta población con un censo de 12 individuos. Un inventario de Pérez Latorre et al. (1996:253) en dicha localidad indica su adscripción fitosociológica, hábitat y abundancia.

\section{Fam. Equisetaceae}

23.- Equisetum ramosissimum Desf., FI. Atlant. 2: 398 (1799)

Marbellí y Rondense. Frecuente. Suelos húmedos sobre cualquier substrato. SaliciPopuletea. 210- $1400 \mathrm{msnm}$. Benahavís (MGC 79510), El Burgo (MGC 86816), Istán (MGC 87093), Parauta (MGC 85945), Ronda (MGC 92222), Yunquera (MGC 87453), Tolox (MGC 87740).

24.-Equisetum telmateia Ehrh. in Hannover. Mag. 21: 287 (1783)

Marbellí y Rondense. Ocasional. Suelos húmedos sobre cualquier substrato. SaliciPopuletea. 600-700 msnm. El Burgo (MGC 89509), Istán (MGC 41747), Yunquera (MGC 87454).

\section{Fam. Polypodiaceae}

25. - Polypodium cambricum subsp. cambricum L., Sp. PI. 1086 (1753)

Marbellí y Rondense. Ocasional. Rupícola, sobre calizas, dolomías, gneis y micaesquistos. Anomodonto-Polypodietea. 340-1000 msnm. Benahavís (MGC 87556), Istán (MGC 89366), Ronda (MGC 87486), Tolox (MGC 87733).

26.- Polypodium interjectum Shivas in J. Linn. Soc., Bot. 58: 29 (1961).

Marbellí. Ocasional. Rupícola, subrupícola (taludes) y epífita en sotobosques de alcornocales y quejigales. Anomodonto-Polypodietea, Quercetea ilicis. 250-1325 msnm. Benahavís (MGC 85057), Igualeja (MGC 90023), Istán (MGC 84807).

Fam. Pteridaceae (Incluidas: Adiantaceae, Sinopteridaceae, Cryptogrammaceae y Hemionitidaceae)

27.- Adiantum capillus-veneris L., Sp. PI. 1096 (1753).

Marbellí, Bermejense y Rondense. Frecuente. Roquedos y taludes rezumantes, sobre todo en calizas y peridotitas. Adiantetea. 390-1100 msnm. Benahavís (MGC 85309), El Burgo (MGC 86832), Istán (MGC 87147), Ronda (MGC 89532), Tolox (MGC 88313). 

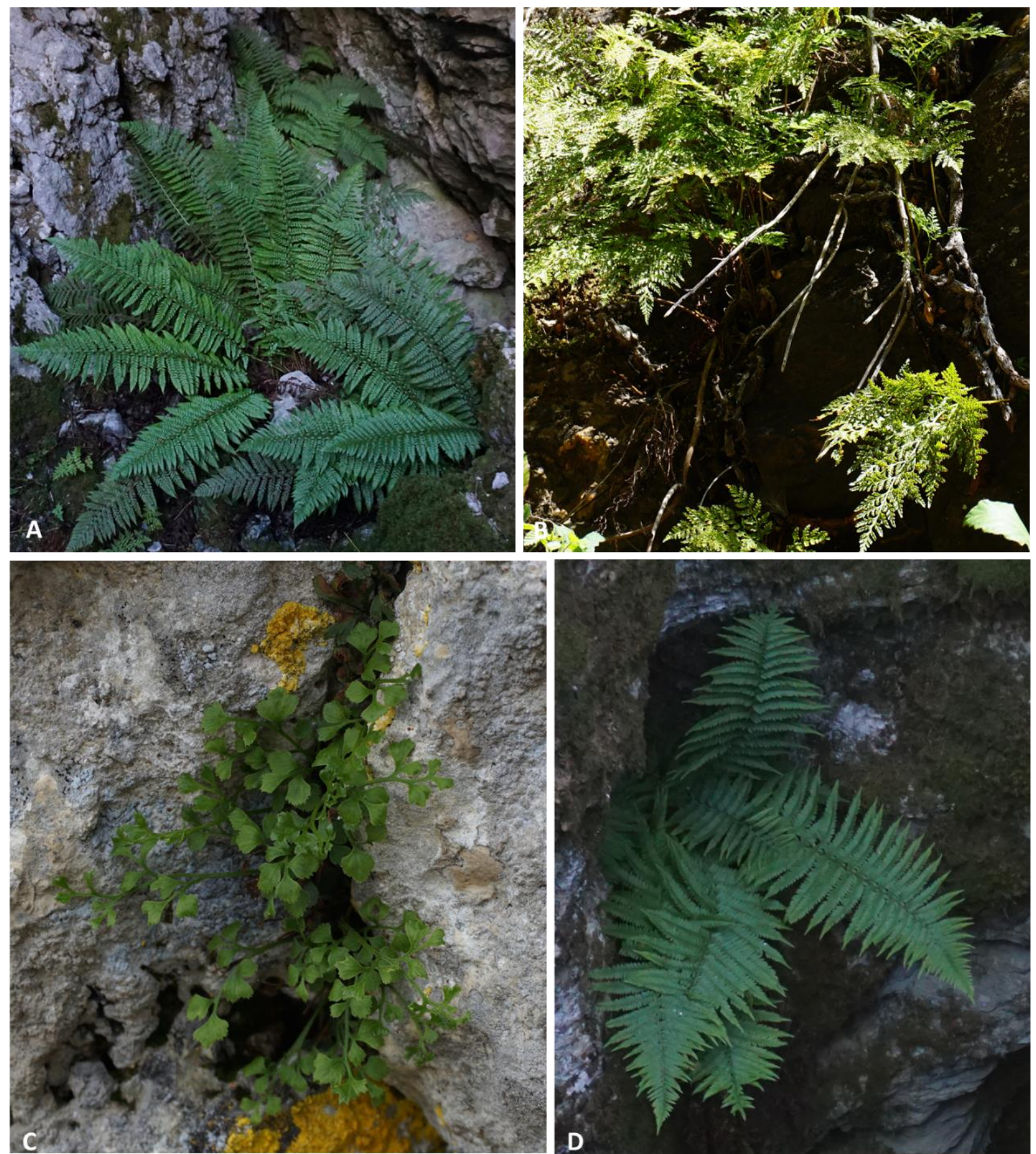

Figura 4. Helechos cuyas poblaciones en el Espacio Natural Sierra de las Nieves representan su límite de distribución en la Península Ibérica: A) Polystichum aculeatum (Puerto de los Pilones, $1750 \mathrm{msnm}$ ); B) Davallia canariensis (Tajos del Mirador, $550 \mathrm{msnm}$ ); C) Asplenium ruta-muraria (Pilar de Tolox, $1750 \mathrm{msnm}$ ); D) Dryopteris filix-mas (Hoyos del Pilar, Sima Honda, 1715 msnm).

Figure 4. Ferns whose populations in the Sierra de las Nieves natural Area represente their distribution limit in the Iberian Peninsula: A) Polystichum aculeatum (Puerto de los Pilones, $1750 \mathrm{msnm}$ ); B) Davallia canariensis (Tajos del Mirador, $550 \mathrm{msnm}$ ); C) Asplenium ruta-muraria (Pilar de Tolox, $1750 \mathrm{msnm}$ ); D) Dryopteris filix-mas (Hoyos del Pilar, Sima Honda, $1715 \mathrm{msnm})$.

28.- Anogramma leptophylla (L.) Link, Fil. Spec. $137(1841)$

Marbellí y Bermejense. Ocasional. Taludes umbríos en substratos ácidos o peridotíticos, ocasionalmente en calizas. AnomodontoPolypodietea. 320-1300 msnm.; Benahavís (MGC 78277), Igualeja (MGC 24071), Istán (MGC 84770), Tolox (MGC88260).
29.- Cosentinia vellea (Aiton) Tod. in Giorn. Sci. Nat. Econ. Palermo 1: 220 (1866). (sensu lato).

Marbellí y Bermejense. Frecuente. Taludes y roquedos en exposiciones soleadas, termófila Asplenietea trichomanis. 280-880 msnm. Benahavís (MGC 78233), Istán (MGC 89385), Tolox (MGC 5360). 
30.- Oeosporangium acrosticum (Balbis) L. Sáez \& P. Aymerich in Orsis, 31:33 (2017)

[ $=$ Cheilanthes acrostica (Balb.) Tod. in Giorn. Sci. Na.Econ. Palermo 1: 215 (1866)]

Marbellí, Bermejense y Rondense. Frecuente. Roquedos expuestos en todo tipo de substratos. Asplenietea trichomanis. 300-1300 msnm. Benahavís (MGC 82459), El Burgo (MGC 87952), Istán (MGC 84737), Ronda (MGC 87489), Tolox (MGC 85435), Yunquera (MGC 90691).

31.- Oeosporangium guanchicum (Bolle) FraserJenk. \& Pariyar in checklist of Indian Pteridophytes, 1: 265 (2016)

[= Cheilanthes guanchica Bolle in Bonplandia 7: 107 (1859)]

Marbellí, Bermejense y Rondense. Frecuente. Roquedos y taludes, rara vez en calizas. Asplenietea trichomanis. 400-1100 msnm. Benahavís (MGC 87602), El Burgo (MGC 87952), Istán (MGC 87694), Tolox (MGC 88257).

32.- Oeosporangium hispanicum (Mett.) FraserJenkins \& Pariyar in checklist of Indian Pteridophytes, 1: 265 (2016)

[= Cheilanthes hispanica Mett. in Abh. Senckenberg. Naturf. Ges. 3: 74 (1859)]

Marbellí y Bermejense. Rara. Roquedos expuestos. Asplenietea trichomanis. 200-820 msnm. Benahavís (MGC 55797), Tolox (MGC 32102).

33.- Oeosporangium $\times$ malacitense (Rasbach \& Reichst.) Arana \& Moreno-Saiz in Phytotaxa 433(3): 191 (2020)

[= Oeosporangium acrosticum $\times$ O. guanchicum]

[= Cheilanthes $\mathrm{x}$ malacitensis Rasbach \& Reichstein in Webbia 35: 261-273 (1982)].

Bermejense. Muy rara. Roquedos. Asplenietea trichomanis. Sierra Palmitera (Igualeja), $1100 \mathrm{msnm}$. (Holotipo en G, cf. Arana \& Moreno Saiz, 2020).

Los únicos datos que tenemos sobre la posible presencia de este taxón en la zona son los del typus: "Sierra Bermeja, serpentinite rocks under trees, $E$ above road from Ronda to San Pedro de Alcántara, ca. $23 \mathrm{~km}$ from Ronda at $1100 \mathrm{~m}$. (cf. Arana \& Moreno Saiz, 2020).

34.- Oeosporangium $x$ tolocense (Rasbach, Reichst. \& Schneller) Arana \& Moreno-Saiz in Phytotaxa 433(3): 192 (2020)

[=Oeosporangium guanchicum $\times$ O. pteridioides]

[= Cheilanthes $x$ tolocensis Rasbach, Reichstein \& Schneller in Webbia 37: 43-62 (1983)].

Bermejense. Muy raro. Roquedos. Asplenietea trichomanis. 300-400 msnm. Tolox (B 20 0125289) (isotypus), Benahavís (MGC 21815).

Las únicas referencias que tenemos de este taxón en la zona son la localidad del typus:" $1.6 \mathrm{~km}$ SW of Tolox and SW of the Casa de Baños on the right (SE) side of the Río de los Caballos" (Arana \& Moreno Saiz, 2020) y de un pliego depositado en el herbario de la Universidad de Málaga (MGC 21815) en el que indica la localidad de Benahavís, a la altura del Madroñal.
35.- Oeosporangium pteridioides (Reichard) Fraser-Jenkins \& Pariyar in checklist of Indian Pteridophytes, 1: 258 (2016)

[ $=$ Cheilanthes maderensis Lowe in Trans. Cambridge Philos. Soc. 6: 528 (1838)].

[= Cheilanthes pteridioides (Reichard) C. Chr., Index Filic.: 178 (1905)]

Marbellí y Bermejense. Rara. Roquedos expuestos. Asplenietea trichomanis. 500-700 msnm. Benahavís (MGC 78224), Istán (MGC 89846), Tolox (MGC 37632),

36.- Oeosporangium tinaei (Todaro) FraserJenkins in checklist of Indian Pteridophytes, 1: 258 (2016)

[= Cheilanthes tinaei Tod. in Giorn. Sci. Nat. Econ. Palermo 1: 217 (1866)]

Marbellí. Muy rara. Taludes. Asplenietea trichomanis. 900 msnm. Istán (MGC 39342).

37.- Paragymnopteris marantae (L.) K.H. Shing in Indian Fern J. 10: 229 (1994)

[=Notholaena marantae (L.) Desv. in J. Bot. Agric. 1: 92 (1813)]

Bermejense. Ocasional. Roquedos, gleras y taludes peridotíticos en situaciones expuestas. Asplenietea trichomanis. 350-1100 msnm. Benahavís (MGC 78299), Istán (MGC 85222), Tolox (MGC 91598). Considerado como serpentinófito obligado (Pérez Latorre et al., 2013).

38.- Pteris vittata L., Sp. Pl.: 1074 (1753).

Marbellí. Rara. Borde de arroyos y taludes rezumantes y térmicos. Adiantetea. 200-450 msnm. Benahavís (MGC 78322), Istán (MGC 84890).

\section{Conclusiones}

Se han localizado 38 taxones en el territorio estudiado (36 especies y subespecies y 2 híbridos). $\mathrm{Si}$ comparamos este número con la riqueza de pteridófitos en otros Espacios Naturales Protegidos cercanos solo es comparable el Parque Natural de los Alcornocales, también con 38 taxones (Cabezudo \& Salvo, 1987; Guerra et al. 2003), en los otros espacios naturales protegidos la diversidad es menor, el Parque Natural de Grazalema con 21 (Aparicio \& Silvestre, 1987) y el Parque Natural de Tejeda, Alhama y Almijara con 30 (Cabezudo et al. 2005). La diversidad pteridológica de la zona de estudio se debe a la posición geográfica que la zona ocupa entre dos de las zonas más ricas en helechos de la Península lbérica como las Sierras Béticas y sierras de Algeciras (Moreno Saiz et al. 2015), enriquecida además por la presencia de una importante superficie de sierras serpentínicas (sierras bermejas).

La diversidad de helechos en número de taxones en los tres subsectores del espacio natural es: Bermejense 16, Rondense 21 y Marbellí 24. El subsector Marbellí presenta 8 taxones exclusivos, el Rondense 9 y el Bermejense 2. Comunes en los tres subsectores son 7 taxones. El subsector Marbellí, el 
más oriental del sector Aljíbico, carece de la gran riqueza pteridológica del resto de los subsectores aljibicos (Pérez Latorre et al., 1996).

La riqueza de taxones no se distribuye de forma homogénea en el territorio, existiendo 3 zonas que destacan por encima del resto (Figura 5): A/ La zona más elevada de la Sierra Blanquilla del Burgo, que acoge en los mismos roquedos a Asplenium azomanes, $A$. ceterach, $A$. hispanicum y $A$. petrarchae. B/ La parte superior de la cuenca del río Guadaiza, zona que alberga todos los helechos de óptimo aljíbico, destacando Asplenium billotii, Athyrium filix-femina, y las únicas poblaciones de Davallia canariensis y Polystichum setiferum del Espacio Natural. C/ Por último, la Meseta del Quejigal de Tolox en la zona superior del Parque Natural Sierra de las Nieves, por encima de los 1700 msnm, donde se refugian toda una serie de taxones relictos cuyas poblaciones aquí son finícolas en su distribución ibérica y SW europea como Asplenium ruta-muraria, Dryopteris filix-mas y Polystichum aculeatum, además de los raros Asplenium csikii, $A$. jessenii y $A$. scolopendrium, entre otros.

El hábitat de roquedos con 24 taxones es el más frecuentemente ocupado por las especies de pteridófitos en el espacio natural estudiado, lo que podría estar en consonancia con el macrobioclima Mediterráneo predominante (Rivas Martínez, 2011) que selecciona especies que se refugian en ese tipo de biotopos. En taludes se localizan 12 especies y 6 en suelos más o menos húmedos. En las simas presentes en el territorio se localizan tres especies, Polystichum aculeatum, Dryopteris filix-mas $y$ Asplenium scolopendrium, taxones nemorales de bosques caducifolios (Querco-Fagetea) que en la actualidad se refugian en estas simas que mantienen las condiciones hoy perdidas en los bosques de Quercus alpestris Boiss., debido al secular adehesamiento y aprovechamiento ganadero.

Destacan en la zona estudiada la presencia de poblaciones finícolas de Davallia canariensis, de distribución macaronésica e ibero-atlántica, o Polystichum aculeatum de óptimo eurosiberiano, esto es debido a que en el Espacio Natural se localizan las representaciones más orientales de la vegetación aljíbica y las formaciones vegetales supra-oromediterráneas más meridionales de la Península Ibérica. En este sentido hay que señalar que tanto las poblaciones de $P$. aculeatum como de Dryopteris filix-mas y Asplenium ruta-muraria del Espacio Natural son las únicas de estas especies en la Cordillera Bética Occidental, lo que da idea de su relictualidad.

Un total de 19 especies son muy raras o raras en el territorio, motivo por lo que el PRUG (Plan Rector de Uso y Gestión) de los dos parques que conformarán el Espacio Natural deberían aplicar algunas medidas de gestión y protección para estas especies. Otras 18 especies son ocasionales o frecuentes. Solo una especie, Asplenium billotii, está legalmente protegida como RPE (Régimen de Protección Especial) en la legislación andaluza, Davallia canariensis y Dryopteris filix-mas están consideradas como VU (Vulnerables) en Andalucía y Asplenium billotii y Polystichum setiferum como NT (Casi Amenazadas).

De Oeosporangium están presentes las 5 especies ibéricas y todas las de la subfam. Cheilanthoideae, al estar presente también Paragymnopteris marantae (Gabriel y Galán et al, 2018).

En la zona de estudio las poblaciones de $A$. trichomanes (s.l.) estrictamente rupícolas, calcícolas, con frondes cortas y aplicadas al roquedo han sido tradicionalmente adscritas a la subsp. pachyrrachys (cf. Salvo, 1990; Pérez Latorre et al., 1998; Cabezudo, 2011). Este taxón es, entre los poliploides del agregado, el que tiene unos caracteres morfológicos mejor definidos (cf. Lovist \& Reichstein, 1980; Sáez, 2000), como frondes aplicadas al substrato, pinnas triangulares netamente más largas que anchas y biauriculadas en la base. Sin embargo, buena parte de las poblaciones de nuestra zona de estudio adscritas a la subsp. pachyrrachys presentan una morfología muy distinta tanto a la del tipo de $A$. csikii (Imagen de los typus consultados on-line en GBIF: $K$ 001092487), como a la diagnosis original de $A$. trichomanes subsp. pachyrrachys. Sin embargo, los caracteres morfológicos de nuestras poblaciones corresponden a la descripción que hace Sáez (2000) de la subsp. hastatum (Christ) Jessen. Sin embargo, y siguiendo también a Sáez (2000), parte de estas poblaciones pudieran pertenecer a la subsp. inexpectans, taxón diploide citado en la Serranía de Ronda (Rasbach et al., 1990). Este problema no es nuevo, Pangua et al. (1989), adjudicaron a la subsp. pachyrrachys, con muchas dudas, material ibérico con caracteres morfológicos similares a los nuestros. Posteriormente Sáez (2000) indicó que estos materiales pudieran corresponder a la subsp. hastatum o a la subsp. inexpectans. Por tanto, es necesario un estudio cromosómico o micromorfológico (longitud de las esporas como carácter diagnóstico entre taxones diploides y tetraploides del agregado) de nuestras poblaciones para aclarar la presencia de los distintos taxones de este grupo. 

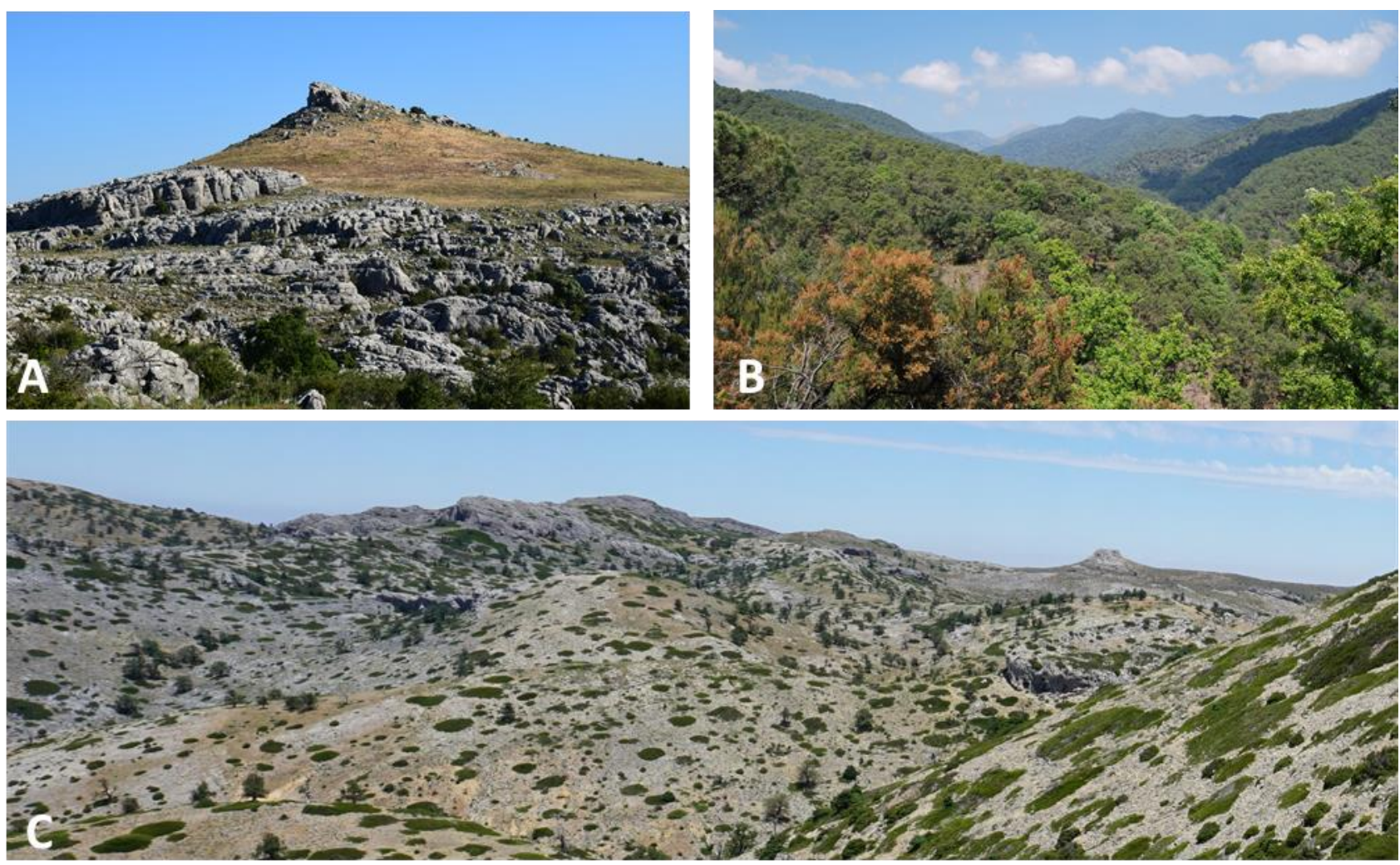

Figura 5. Tres de los enclaves más singulares, por los helechos que albergan, del Espacio Natural Sierra de las Nieves: A) Sierra Blanquilla, alrededores del cerro del Viento; B) Parte alta de la Cuenca del río Guadaiza, desde las Maquinas a las casas del Daidín; C) La zona a mayor altitud del Espacio Natural Sierra de las Nieves, Meseta del Quejigal de Tolox. Figure 5. Three of most unique places, for its ferns, of the Sierra de las Nieves Natural Area: A) Sierra Blanquilla surroundings of Cerro del Viento; B) Upper part of the Guadaiza river basin, from The Machine to Daidin's Houses; C) The highest altitude area of Sierra de las Nieves Natural Area, Plateau of Quejigal de Tolox.

\section{Agradecimientos}

A Rafael Haro, Director del Parque Natural Sierra de las Nieves, por su ayuda en la localización de la población de Polystichum aculeatum y las facilidades dadas para acceder a todas las zonas del Espacio Natural. A Oscar Gavira por sus comentarios y sugerencias sobre los helechos en general y el agregado de Asplenium trichomanes en particular. A José García, conservador del herbario MGC, por su diligencia en todos los temas referidos a la conservación e informatización de todo el material recolectado en la sierra. A Noelia Hidalgo por facilitarnos el mapa de la Figura 1. A Marta Recio por su ayuda en el estudio de las esporas del género Cystopteris.

Trabajo realizado en el marco del Convenio de Colaboración firmado en Diciembre de 2018 entre la Consejería de Medio Ambiente y Ordenación del Territorio de la Junta de Andalucía y la Universidad de Málaga para el estudio de la Flora y Vegetación del futuro Parque Nacional Sierra de las Nieves.

\section{Bibliografía}

Aparicio, A. \& Silvestre, S. (1987). Flora del Parque natural de la Sierra de Grazalema. Junta de Andalucía. Sevilla.
Arana, M.D. \& Moreno Saiz, J.C. (2020). New combinations and typification of Mediterranean Oeosporangium (Pteridaceae: Cheilanthoideae). Phytotaxa, 433 (3), 190-194.

Blanca, G., Cabezudo, B., Cueto M., Morales Torres, C. \& Salazar, C. (2011). Flora vascular de Andalucía Oriental, 2a edición. Universidades de Granada, Almería, Jaén y Málaga, Granada.

Boissier, E. (1839-1845). Voyage Botanique dans le Midi de l'Espagne Pendant l'année 1837. París, Francia.

Cabezudo, B. (2011). Pteridofitos. In Blanca, G., Cabezudo, B., Cueto M., Morales Torres, C. \& Salazar, C. (Eds.). Flora vascular de Andalucía Oriental, 2a edición. Universidades de Granada, Almería, Jaén y Málaga, Granada.

Cabezudo, B., Pérez Latorre, A., Navas, P., Navas, D. \& Gil, Y. (1998). Parque Natural Sierra de las Nieves: Cartografía y evaluación de la flora y vegetación. Informe técnico. Junta de Andalucía Sevilla.

Cabezudo, B., Pérez Latorre, A.V., Navas, D., Gavira, O. \& Caballero, G. (2005). Contribución al conocimiento de la flora del Parque Natural de las Sierras Tejeda, Almijara y Alhama (MálagaGranada, España). Acta Botanica Malacitana 30, 55-109. 
Cabezudo, B., Rivera, J. \& Salvo, A.E. (1983). Cystopteris diaphana (Bory) Blasdell. Lagascalia, 11, 104-105.

Cabezudo, B. \& Salvo, A.E. (1987). Pteridofitos. In Valdés, B., Talavera S. y Fernández Galiano E. (Eds.). Flora vascular de Andalucía Occidental. Ketres Editora S.A. Barcelona.

Cabezudo, B. \& Talavera, S. (Coord.). (2005). Lista roja de la flora vascular de Andalucía. Junta de Andalucía. Sevilla.

Cabezudo, B. \& Trigo, M. M. (2004). Pteridófitos (helechos y plantas afines). In Blanca-López, G \& Ortega-Díaz, A. (Coord.). Proyecto Andalucía, Naturaleza vol. XXI, Botánica II (pp. 315-374). Sevilla.

Castroviejo, S. et al., (Eds.). (1986). Flora Iberica vol. 1, Lycopodiaceae-Papaveraceae. Real Jardín Botánico,CSIC, Madrid.

Delgado Vazquez, A.J. \& Plaza Arregui, L. (2010). Helechos amenazados de Andalucía. Consejería de Medio Ambiente, Junta de Andalucía.

Ekrt, L. \& Štech, M. (2008). A morphometric study and revision of the Asplenium trichomanes group in the Czech Republic. Preslia, 80, 325-347.

Fraser-Jenkins, C.R., Smith, A.R. \& Windhand, M.D. (2017). Proposal to reject the name Allosorus (Pteridaceae). Taxon, 66 (2), 517-518.

Gabriel y Galán, J. M., Molino, S., De la Fuente, P. \& Seral, A. (2018). Novedades para la pteridoflora ibérica en el contexto de un nuevo sistema para las plantas vasculares sin semilla. Botanica Complutensis, 42, 69-81.

Gavira, O. \& Pérez Latorre, A.V. (2003). Aproximación al catálogo florístico del Valle del río Genal (Serranía de Ronda, Málaga, España). Anales de Biología, 25, 113-161.

Guerra, J., Cano, M.J., Pérez Latorre, A.V, Ros, R.M. \& Cabezudo, B. (2003). Flora briopteridofitica de los bosques lauroides de Rhododendron ponticum $\mathrm{L}$. del Parque Natural de los Alcornocales. (Cádiz-Málaga, España). Acta Botanica Malacitana, 28, 19-36.

Guerra, J., Gallego, M.T., Jimenez, J.A., Cano, M.J., Casimiro-Soriguer, F. Pérez Latorre, A.V. \& Cabezudo, B. (2018). Flora Briofítica del Espacio Natural Sierra de las Nieves y su entorno (Málaga, España). Ed. Universidad de Murcia.

Laza Palacios, M. (1940). Flórula farmacéutica malacitana. Anales de la Real Academia de Farmacia, 6, 165- 214.

Liu, H.-M., Rusell, S.R., Vogel, J. \& Scheneider, J. (2018). Inferring the potential of plastid DNA based identification of derived ferns: a case study on the Asplenium trichomanes aggregate in Europe. Plant Systematics and Evolution, 304,1009-1022.

Lovis, J.D. \& Reichstein, T. (1985). Asplenium trichomanes subsp. pachyrrachys (Aspleniaceae, Pteridophyta) and a note on the typification of $A$. trichomanes. Willdenowia, 15, 187-201.

Morales Abad, M.J. (1990). Adiciones, Mapa 103. Fontqueria 28, 69.

Moreno Saiz, J.M., Pataro, L. \& Pajarón Sotomayor, S. (2015). Atlas de los pteridófitos de la
Península Ibérica e Islas Baleares. Acta Botanica Malacitana, 40, 5-54.

Murphy, R.J. \& Rumsey, F.J. (2005). Cystopteris diaphana (Bory) Blasdell (Woodsiaceae) - an overlooked native new to the British Isles? Watsonia, 25, 255-263.

Nieto Caldera, J.M., Pérez Latorre, A.V., Cabezudo, B., Boyero Gallardo, J.R., Escamez Pastrana, J.M. \& Salvo Tierra, E. (1987). Aportación al catálogo pteridoflorístico de la provincia de Málaga. Acta Botanica Malacitana, 13, 375-377.

Pangua, E., Prada, C., \& Marquina, A. (1989). Las subespecies de Asplenium trichomanes L. en la Península Ibérica e Islas Baleares. Botanica Complutensis, 14, 87-108.

Pérez Latorre, A.V., Galán de Mera, A., Dell, U. \& Cabezudo, B. (1996). Fitogeografía y vegetación del Sector Aljíbico (Cádiz-Málaga, España). Acta Botanica Malacitana, 21, 241-267.

Pérez Latorre, A.V., Hidalgo-Triana, N \& Cabezudo, B. (2013). Composition, ecology and conservation of the south -lberian serpentine flora in the context of the Mediterranean basin. Anales del Jardín Botánico de Madrid, 70(1), 62-71.

Pérez Latorre, A.V., Hidalgo Triana, N. \& Cabezudo, B. (2019). Mapa biogeográfico de la provincia de Málaga. Universidad de Málaga y Diputación de Málaga.

Pérez Latorre, A., Navas, P., Navas, D., Gil Y. \& Cabezudo, B. (1998). Datos sobre la flora y vegetación de la Serranía de Ronda (Málaga, España). Acta Botanica Malacitana, 23, 149-191.

Prada, C. (1986). Cystopteris Bernh. In S. Castroviejo et al. (eds.), Flora iberica I. Lycopodiaceae-Papaveraceae: 115-121. Real Jardín Botánico, C.S.I.C., Madrid.

Rasbach, H., Rasbach, K., Reichstein, T. \& Bennert, H.W. (1990). Asplenium trichomanes subsp. coriaceifolium, a New Subspecies and two new intraspecific hybrids of the $A$. trichomanes Complex (Aspleniaceae, Pteridophyta). I. Nomenclature and Typification. Willdenowia, 19, 471-474.

Rivas Goday, S. (1968). Algunas novedades fitosociológicas de España Meridional. Collectanea Botanica, 7, 997-1031.

Rivas Martínez, S. (2011). Mapa de series, geoseries y geopermaseries de vegetación de España. Memoria del Mapa de Vegetación Potencial de España. Parte 1. Itinera Geobotanica, 18(1), 5-424.

Sáez, LI. (2000). El complex d'Asplenium trichomanes L. (Aspleniaceae, Pteridophyta) al nord-est de la península Ibèrica. Orsis, 15, 27-43.

Sáez, LI., Vazquez, V.M., Cires, E. \& Fernandez Prieto, J.A. (2020). Otra vez sobre el nombre a usar para los helechos de los géneros Cheilanthes (Allosorus) y Cryptogramma. In Fernández Prieto, J.A, V.M. Vázquez, A. Bueno, H.S. Nava, L. Carlón y E. Cires (Eds.). Notas corológicas, sistemáticas y nomenclaturales para el catálogo de la Flora Vascular del Principado de Asturias. IV. Naturalia Cantabricae 8 Especial (2), 61-113. 
Federico Casimiro-Soriguer Solanas et al. La pteridoflora del Espacio Natural Sierra de las Nieves.

Salvo, A.E. (1990). Guía de Helechos de la Península Ibérica y Baleares. Ediciones Pirámide, Madrid.
Salvo, A.E. \& Cabezudo, B. (1984). Lista comentada de los pteridófitos de Andalucía. Acta Botanica Malacitana, 9, 133-146. 